\title{
Prevention of antipsychotic side effects in elderly populations.
}

\author{
Mary V Seeman* \\ Department of Psychiatry, University of Toronto, Toronto, Ontario, Canada
}

\begin{abstract}
The elderly, especially women, are at risk for severe side effects from antipsychotic medication. There are a number of reasons for this. This paper lists the reasons, the nature of the effects, and provides clinical recommendations for prevention and treatment.
\end{abstract}

Accepted on 03 February 2018

\section{Introduction}

Severe mental illnesses such as schizophrenia tend to start early in life and continue throughout adulthood and into old age. Some symptoms improve with age while others remain constant or get worse. There is considerable variability in the progression or plateauing of illness severity over time. The core treatment for schizophrenia is antipsychotic medication, although the choice of an optimal drug regimen varies with patient preference, co-morbidities, adverse drug effects, and interactions with co-administered drugs. In general, guidebook antipsychotic doses for schizophrenia are too high for the elderly; most investigators recommend a gradual reduction of dosages in older age [1]. This is because liver and kidney function deteriorates with age and body composition changes, affecting drug distribution, metabolism, and elimination. There are also important pharmacodynamics changes that take place in the sensitivity of neurotransmitter receptors in the brain, the protein receptors with which the drugs interact.

\section{Pharmacokinetics and Pharmacodynamics}

With respect to pharmacokinetics, Castberg et al. [2] have investigated serum concentrations of second generation antipsychotics (clozapine, olanzapine, risperidone and quetiapine) in relation to age in a large sample of almost 12,000 patients. They found that age exerted a significant impact on the concentrations of all four drugs. When a person reaches the age of 80 , dose-adjusted concentrations double those at age 40 . By age 90, they become two-to-three-fold higher. There are known differences among the drugs, with clozapine showing the largest increased concentrations with age, and olanzapine showing the smallest.

With respect to the pharmacodynamics of antipsychotic drugs on the dopamine D2 receptor, in young adults, the therapeutic window of striatal dopamine D2 receptor occupancy as measured by positron tomography is between $65 \%$ and $85 \%$ [3]. Below $65 \%$ occupancy, there is no antipsychotic effect; above $80 \%-85 \%$ occupancy, the emergence of extrapyramidal side-effects outweighs the antipsychotic benefit. Recent studies suggest that, for individuals over 50 years of age, the therapeutic window is lower, located somewhere between $50 \%$ and $60 \%[4,5]$. With respect to the emergence of hyperprolactinemia, the threshold for which is $73 \%$ of occupied
D2/D3 receptors in the pituitary gland of young adults, that percentage drops to $66 \%$ occupancy in older individuals [6].

In other words, in older age, relatively low doses of antipsychotic medication suffice for symptom control, and adverse side effects can, and often do, emerge at standard adult dosages [7]. The specifics of the side effects differ with age [8]. In the paediatric population, the main side effects are behavioural (e.g. inattention, sedation); in the adult population, they are mainly metabolic (e.g. obesity, diabetes, metabolic syndrome); in the over 65 population, they are mainly neurologic (e.g. extrapyramidal, postural and cognitive).

\section{Neurologic Effects}

Tardive dyskinesia (TD), a movement disorder that involves involuntary choreoathetoid repetitive movements such as chewing and tongue protrusions, is a prevalent adverse effect of antipsychotic medication, especially prevalent in the elderly and usually associated with first generation antipsychotics (AP) more than with newer generation APs. While the mean prevalence of TD in the population who take APs is $20-25 \%$, that percentage climbs steeply with every year of added age [9].

Another neurological adverse effect of APs is postural instability. This disturbance in balance, especially since it often occurs in conjunction with extrapyramidal symptoms such as parkinsonism, akathisia and tardive dyskinesia and sometimes also with cognitive impairment, sedation, orthostatic change in blood pressure and cardiac arrhythmia (all among the relatively common adverse effects of APs), markedly increases the risk of falling and sustaining fractures [10-12]. Hip fractures are particularly dangerous in the elderly and are associated with increased mortality.

Sedation induced by AP drugs is important not only in the context of potentially dangerous falls in the elderly but also because it can lead to respiratory compromise and to fatal kitchen accidents or road accidents [13]. The fact that APs also lead to obesity, which, in turn, strongly contributes to sleep apnea and consequent daytime sleepiness, adds to the prevalence of AP-induced sedation [14].

Cognitive impairment is associated with severe mental illness and can be aggravated by APs, especially in old age. The AP induction of cognitive disturbance is mediated by 
anticholinergic effects inherent in some APs more than in others and, therefore, varies not only with the prescribed dose but also with the specific agent used [15]. It is made worse by the use of adjunctive anticholinergic medications often prescribed to counteract the extrapyramidal side effects of APs [16]. The anticholinergic burden borne by elderly patients is further increased by the frequent addition of antihistamines or sedating antidepressants to the drug regimen [17].

\section{Effects of Hyperprolactinemia}

Hyperprolactinemia induced by APs suppresses the reproductive endocrine axis, with consequent loss of bone mineral density [18]. The use of APs is, as a consequence, strongly associated with bone fractures, with hip fractures constituting the greatest risk to life [19-21]. There is also the reported possibility, although this is highly controversial, that high prolactin levels induced by APs can contribute to the risk of breast and prostate cancer [22-25].

Cardiovascular Effects

Cardiovascular adverse effects, such as postural hypotension leading to faintness [26] and tachycardia (overly rapid heartbeat) [27] are commonly associated with the use of antipsychotics. More serious side effects are arrhythmias leading to sudden death, caused by blockade of cardiac potassium channels and prolongation of the QT interval [28]. Clozapine use has been associated with death from myocarditis and cardiomyopathy [29] Aripiprazole, a partial dopamine agonist, can induce hypertension [30]. The weight gain associated with most APs increases the risk for type 2 diabetes and hyperlipidaemia, which, in turn, induce heart complications. In general, APs must be used with caution in the elderly, for fear of fatal cardiac complications [31,32]. Antipsychotic drugs also exert direct and indirect effects on the vascular system, leading to complications such as venous and pulmonary thromboembolism [33-35]. As a result of this and of the increased risk for atrial fibrillation [36], there is a high prevalence of cerebrovascular complications in the elderly taking APs [37].

\section{Other Important Adverse Effects}

There is an association between APs and pneumonia in the elderly [38,39]. Urinary disease is also reportedly increased [40,41], as is electrolyte imbalance [42] and hypothermia [43]. In addition, APs can induce cataracts that interfere with vision [44] and gastrointestinal hypo motility, which can interfere with the absorption of drugs [45] and, thus, make them less effective.

\section{Interactions}

A cohort study of AP use among over 7 thousand persons aged 65 years or over in a veterans' geriatric service in Australia found that, at a median age of 86 years, 5 percent of veterans received more than one antipsychotic at a time [46]. An Italian study found frequent adverse interactions in the elderly; as many as 5 adverse interactions were found for every patient in the study [47]. A US study of over 3000 adults aged 70-79 found that over one-third were suffering from at least one type of drug interaction [48]. Polypharmacy is common and adverse interactions are common.

\section{Mortality Risk}

Because of cerebrovascular events, arrhythmias, venous thromboembolism, pulmonary embolism, and aspiration pneumonia, antipsychotics constitute a greater mortality risk in older adults than any other psychotropic medication. Metabolic effects (obesity, lipid disturbances and altered glucose homeostasis) and accidents due to light-headedness, sedation, and cognitive difficulties combined with osteoporosis lead to falls and hip fractures, frequently fatal in older age [49].

\section{Gender Differences}

Whereas both women and men show the impact of APs as they age, there are notable gender differences. With advancing age, women's blood concentrations of APs are 20-30\% higher than those of men prescribed the same dose [2]. Our group found the duration of the postmenopausal period to be significantly and negatively associated with antipsychotic response in women [50]. Antipsychotic response declines sharply in women after the loss of estrogen after menopause and, later, in both sexes after andrenopause. Because this means that the drugs become progressively less effective in relieving psychotic symptoms, their dose is increased, leading to high blood concentrations and an intensification of adverse effects [51].

\section{Recommendations for Physicians Treating Elderly Patients with Severe Mental Illness}

On the basis of this brief review of the evidence, several clinical recommendations can be made, borrowed from Pretorius et al. [52]. The Pretorius et al. paper refers to the use of all medications, not only to psychiatric ones. The recommendations are especially relevant to patients with severe mental illness who, for several reasons, tend to receive substandard care by the medical profession. Because of apathy and cognitive disturbances, they do not, generally, advocate for themselves; many are estranged from family members who would otherwise advocate for them; many distrust their doctors and avoid visits. Many individuals with SMI alienate their care providers because they miss appointments, come late, do not adhere to diet, substance use, or physical activity regimens, do not take their medications as prescribed and often create disturbances in the waiting room. Patient-doctor communication is often difficult. For all these reasons, the following recommendations are particularly important.

A) As patients with SMI age and renal and hepatic function deteriorates, it is important to review all prescriptions with them repeatedly, and gradually reduce dosages. B) Whenever a patient is re-admitted to hospital for an acute episode of psychosis, AP doses tend to increase. It is critically important to remember to reduce these doses gradually to a stable maintenance dose after hospital discharge. C) It is always safer to treat adverse effects of drugs by lowering doses than by 
adding another drug to counter the effects of the first one. D) Many patients take over-the-counter or herbal oral preparations as well as functionally active ointments and inhalants. It is important to inquire about these because they can interact with antipsychotic drugs to produce adverse effects. E) When a prescribed drug is no longer effective, it is tempting to raise the dose. Ineffectiveness, however, may be due to the patient's not taking it. Alternatively, symptoms may result from withdrawal and rebound reactions as patients periodically start and stop medication. Non-adherence to a drug regimen is a prevalent phenomenon in the SMI population that needs to be periodically checked. Sometimes the drug is not effective because it is not being absorbed. A parenteral route of entry may prove effective. For many older people, low-level psychotic symptoms may be more tolerable than the side effects that come with higher doses. F) AP drugs have different therapeutic windows or effective dose ranges. Those with wide windows are safest for elderly patients. G) When initiating medication in elderly patients, "starting low and going slow" is good advice. H) More than one new medication should not be started at the same time. I) Since elderly patients always have more than one co-morbidity, it is important to liaise with all treating physicians. J) Medication regimens need to be frequently reviewed and those that are ineffective removed. All changes need to be instituted slowly. K) Women are more at risk for adverse effects than men. L) Elderly patients should be seen frequently, home visits often being necessary in this population. M) Because patients with severe mental illness may have no close relatives or friends, it is often essential to link them with community services and visiting nurses or case workers to assist in the monitoring of health and habits.

\section{Conclusion}

The elderly are at serious risk for the adverse effects of antipsychotic medication, a risk that is preventable.

\section{References}

1. Tsuboi T, Suzuki T, Uchida H. A tipping point in drug dosing in late-life schizophrenia. Curr Psychiatry Rep. 2011;13(3):225-33.

2. Castberg I, Westin AA, Skogvoll E, et al. Effects of age and gender on the serum levels of clozapine, olanzapine, risperidone and quetiapine. Acta Psychiatr Scand. 2017;136(5):455-64.

3. Farde L, Wiesel FA, Halldin C, et al. Central D2-dopamine receptor occupancy in schizophrenic patients treated with antipsychotic drugs. Arch Gen Psychiatry. 1988;45(1): 71-6.

4. Graff-Guerrero A, Rajji TK, Mulsant BH, et al. Evaluation of antipsychotic dose reduction in late-life schizophrenia a prospective dopamine D2/3 receptor occupancy study. J Am Med Assoc Psychiatry. 2015;72(9):927-34.

5. Reeves S, Eggleston K, Cort E, et al. Therapeutic D2/3 receptor occupancies and response with low amisulpride blood concentrations in very late-onset schizophrenia-like psychosis (VLOSLP). Int J Geriatr Psychiatry. 2018;33(2): 396-404.
6. Iwata Y. Threshold of dopamine D2/3 receptor occupancy for hyperprolactinemia in older patients with schizophrenia. J Clin Psychiatry. 2016;77(12):e1557-63.

7. Suzuki T, Remington G, Uchida H, et al. Management of schizophrenia in late life with antipsychotic medications. A qualitative review. Drugs Aging. 2011;28(12):961-80.

8. Sagreiya H, Chen YR, Kumarasamy NA, et al. Differences in antipsychotic-related adverse events in adult, pediatric and geriatric populations. Cureus. 2017;9(2):e1059.

9. Goldberg RJ. Tardive dyskinesia in elderly patients-an update. J Am Med Dir Assoc. 2002;3(3):152-61.

10. de Groot MH, van Campen JP, Moek MA, et al. The effects of fall-risk-increasing drugs on postural control: A literature review. Drugs Aging. 2013;30(11):901-20.

11. Huang AR, Mallet L, Rochefort CM, et al. Medicationrelated falls in the elderly: Causative factors and preventive strategies. Drugs Aging. 2012;29(5):359-76.

12. Milos V, Bondesson $\AA$, Magnusson M, et al. Fall riskincreasing drugs and falls: A cross-sectional study among elderly patients in primary care. BMC Geriatr. 2014;14:40.

13. Miller DD. Atypical antipsychotics: Sleep, sedation and efficacy. Prim Care Companion J Clin Psychiatry. 2004;6(Suppl 2):3-7.

14. Linselle M, Sommet A, Bondon-Guitton E, et al. Can drugs induce or aggravate sleep apneas? A case-noncase study in VigiBase ${ }^{\circledR}$, the WHO pharmacovigilance database. Fundamentals of Clinical Pharmacology. 2017;31(3):359-66.

15. Ang MS, Abdul Rashid NA, Lam M, et al. The impact of medication anticholinergic burden on cognitive performance in people with schizophrenia. J Clin Psychopharmacol. 2017;37(6):651-6.

16. Pristed SG, Correll CU, Nielsen J. Frequency and correlates of anticholinergic use among patients with schizophrenia in Denmark: A Nation-wide pharmacoepidemiological study. Psychiatry Res. 2017; 255:198-203.

17. Montastruc F, Benevent J, Touafchia A, et al. Atropinic (anticholinergic) burden in antipsychotic-treated patients. Fundamentals of Clinical Pharmacology. 2018;32(1): 114-9.

18. O’Keane V. Antipsychotic-induced hyper-prolactinaemia, hypogonadism and osteoporosis in the treatment of schizophrenia. J Psychopharmacol. 2008;22(2 Suppl): 70-5.

19. Bakken MS, Schjøtt J, Engeland A, et al. Antipsychotic drugs and risk of hip fracture in people aged 60 and older in Norway. J Am Geriatr Soc. 2016;64(6):1203-9.

20. Bolton JM, Morin SN, Majumdar SR, et al. Association of mental disorders and related medication use with risk for major osteoporotic fractures. $\mathrm{J}$ Am Geriatr Soc. 2017;74(6):641-8.

21. Lee SH, Hsu WT, Lai CC, et al. Use of antipsychotics increases the risk of fracture: A systematic review and meta-analysis. Osteoporos Int. 2017;28(4):1167-78.

22. Jacobson EM, Hugo ER, Borcherding DC, et al. Prolactin in breast and prostate cancer: Molecular and genetic perspectives. Discov Med. 2011;11(59)315-24. 
23. Reutfors $\mathrm{J}$, Wingård $\mathrm{L}$, Brandt $\mathrm{L}$, et al. Risk of breast cancer in risperidone users: A nationwide cohort study. Schizophr Res. 2017;182:98-103.

24. Tworoger SS, Eliassen AH, Zhang X, et al. A 20 year prospective study of plasma prolactin as a risk marker of breast cancer development. Cancer Res. 2013;73(15): 4810-9.

25. Wang $\mathrm{M}, \mathrm{Wu} \mathrm{X}$, Chai $\mathrm{F}$, et al. Plasma prolactin and breast cancer risk: A meta-analysis. Sci Rep. 2016;6:25998.

26. Luukkonen A, Tiihonen M, Rissanen T, et al. Orthostatic hypotension and associated factors among home care clients aged 75 years or older - A population-based study. J Nutr Health Aging. 2018;22(1):154-8.

27. Nilsson BM, Edström O, Lindström L, et al. Tachycardia in patients treated with clozapine versus antipsychotic long-acting injections. Int Clin Psychopharmacol. 2017; 32(4):219-24.

28. Vandael E, Vandenberk B, Vandenberghe $J$, et al. Development of a risk score for QTc-prolongation: The RISQ-PATH study. Int J Clin Pharmacol. 2017; 39(2): 424-32.

29. Khana AA, Ashraf A, Baker D, et al. Clozapine and incidence of myocarditis and sudden death - Long term Australian experience. Int J Cardiol. 2017;238:136-9.

30. Seven H, Ayhan MG, Kürkcü A, et al. Aripiprazoleinduced asymptomatic hypertension: Case report. Psychopharmacol Bull. 2017;47(2):53-6.

31. Polcwiartek C, Kragholm K, Schjerning O, et al. Cardiovascular safety of antipsychotics: A clinical overview. Expert Opin Drug Saf. 2016;15(5):679-88.

32. Szmulewicz AG, Angriman F, Pedroso FE, et al. Longterm antipsychotic use and major cardiovascular events: A retrospective cohort study. J Clin Psychiatry. 2017;78(8):e905-12.

33. Ferraris A, Szmulewicz AG, Vazquez FJ, et al. Antipsychotic use among adult outpatients and venous thromboembolic disease: A retrospective cohort study. J Clin Psychopharmacol. 2017;37(4):405-11.

34. Kahl KG, Westhoff-Bleck M, Krüger THC. Effects of psychopharmacological treatment with antipsychotic drugs on the vascular system. Vascul Pharmacol. 2017;96-98:11-8.

35. Letmaier M, Grohmann R, Kren C, et al. Venous thromboembolism during treatment with antipsychotics: Results of a drug surveillance programme. World J Biol Psychiatry. 2017;28:1-12.

36. Chou RH, Lo LW, Liou YJ, et al. Antipsychotic treatment is associated with risk of atrial fibrillation: A nationwide nested case-control study. Int J Cardiol. 2017;227:134-40.

37. Hsu WT, Esmaily-Fard A, Lai CC, et al. Antipsychotics and the risk of cerebrovascular accident: A systematic review and meta-analysis of observational studies. J Am Med Dir Assoc. 2017;18(8):692-9.

38. Herzig SJ, LaSalvia MT, Naidus E, et al. Antipsychotics and the risk of aspiration pneumonia in individuals hospitalized for nonpsychiatric conditions: A cohort study. J Am Geriatr Soc. 2017;65(12):2580-6.
39. Sultana J, Calabró M, Garcia-Serna R, et al. Biological substantiation of antipsychotic-associated pneumonia: Systematic literature review and computational analyses. PLoS One. 2017;12(10):e0187034.

40. Hsu WY, Muo CH, Ma SP, et al. Association between schizophrenia and urinary incontinence: A populationbased study. Psychiatry Res. 2017; 248:35-9.

41. van Strien AM, Souverein PC, Keijsers CK, et al. Antipsychotic drug use associated with urinary tract infections in older women. Maturitas. 2017;98:46-50.

42. Yang HJ, Cheng WJ. Antipsychotic use is a risk factor for hyponatremia in patients with schizophrenia: A 15 year follow-up study. Psychopharmacology(Berl). 2017;234(5): 869-76.

43. Seeman MV. Antipsychotics, hypothermia, undressing and death. Glob J Med Clin Rep. 2017;4(1):1-3.

44. Chu CS, Chou PH, Chen YH, et al. Association between antipsychotic drug use and cataracts in patients with bipolar disorder: A population-based, nested case-control study. J Affect Disord. 2017; 209:86-92.

45. Chen HK, Hsieh CJ. Risk of gastrointestinal hypomotility in schizophrenia and schizoaffective disorder treated with antipsychotics: A retrospective cohort study. Schizophr Res. 2017.

46. Kalisch Ellett LM, Pratt NL, Kerr M, et al. Antipsychotic polypharmacy in older Australians. 2017.

47. Allegri N, Rossi F, Del Signore F, et al. Drug prescription appropriateness in the elderly: An Italian study. Clin Interv Aging. 2017; 12:325-33.

48. Hanlon JT, Perera S, Newman AB, et al. Potential drugdrug and drug-disease interactions in well-functioning community-dwelling older adults. J Clin Pharm Ther. 2017;42(2):228-33.

49. Chiesa D, Marengoni A, Nobili A, et al. Antipsychotic prescription and mortality in hospitalized older persons. Psychogeriatrics. 2017;17(6):397-405.

50. González-Rodríguez A, Catalán R, Penadés R, et al. Antipsychotic response worsens with postmenopausal duration in women with schizophrenia. J Clin Psychopharmacol. 2016;36(6):580-7.

51. Mathews SB, Epperson CN. Neuropsychiatric disorders among aging women: assessing risk factors and tailoring treatment. Curr Behav Neurosci Rep. 2015;2(4):246-55.

52. Pretorius RW, Gataric G, Swedlund SK, et al. Reducing the risk of adverse drug events in older adults. Am Fam Physician. 2013;87(5):331-6.

\section{*Correspondence to:}

Mary V Seeman

University of Toronto

Ontario, Canada

E-mail: mary.seeman@utoronto.ca 